\title{
CHITOSAN COATED IRON-OXIDE NANOPARTICLE SYNTHESIS USING A DROPLET BASED MICROFLUIDIC REACTOR
}

\author{
Malik Abdul Wahab ${ }^{1}$ and E. Yegan Erdem ${ }^{1,2 *}$ \\ ${ }^{1}$ Mechanical Engineering Department, Bilkent University, Ankara 06800, Turkey \\ ${ }^{2}$ UNAM (National Nanotechnology Research Center), Ankara 06800, Turkey
}

\begin{abstract}
A microfluidic reactor for the synthesis of chitosan coated iron-oxide nanoparticles is described. Tapered double Tjunction is used to generate droplets of reactants (iron chloride solution and chitosan solution) which were merged using a pillar array. Third reactant ammonia solution is introduced after the mixing of already merged droplets. Ammonia solution initiates the reaction and precipitates are collected at the outlet. Transmission electron microscope (TEM) imaging along with Fourier transform infrared spectroscopy (FTIR) is used to characterize the nanoparticles. These nanoparticles have applications in nano-medicine where they can be used as drug carriers.
\end{abstract}

\section{KEYWORDS}

Microfluidics, Droplet based flow, Nanoparticle, Nanoparticle generation, Microreactors, Nano-drug, Chitosan, Iron-oxide.

\section{INTRODUCTION}

Nanoparticles have been the focus of researchers in the past few decades because of their unique structural, mechanical, thermal, optical and chemical properties [1]. Several techniques have been employed for nanoparticle synthesis; both by using batch-wise production as well as microfluidic platforms. Droplet-based microfluidic platforms have many advantages over conventional batchwise production which includes increased control over reaction conditions such as mixing time, concentration and temperature as well as handling of small volumes and improved reaction kinetics [2].

This work is the first study where chitosan coated magnetic iron-oxide nanoparticles was synthesized inside a microfluidic device. These particles have a high potential to be used in directed drug delivery applications. Formerly chitosan coated nanoparticles were synthesized by batch-wise techniques [3, 4]. In this work we demonstrate the synthesis of chitosan coated iron-oxide nanoparticles in a droplet-based microfluidic device. We generate droplets from two streams of reagents in an alternating way and use a pillar array to merge them. Later, we merge the third reagent to initiate the precipitation of iron oxide. For maintaining an alternating droplet generation, tapered channel geometry is used [5].

\section{EXPERIMENTAL}

Figure 1 shows the layout of the microfluidic device.

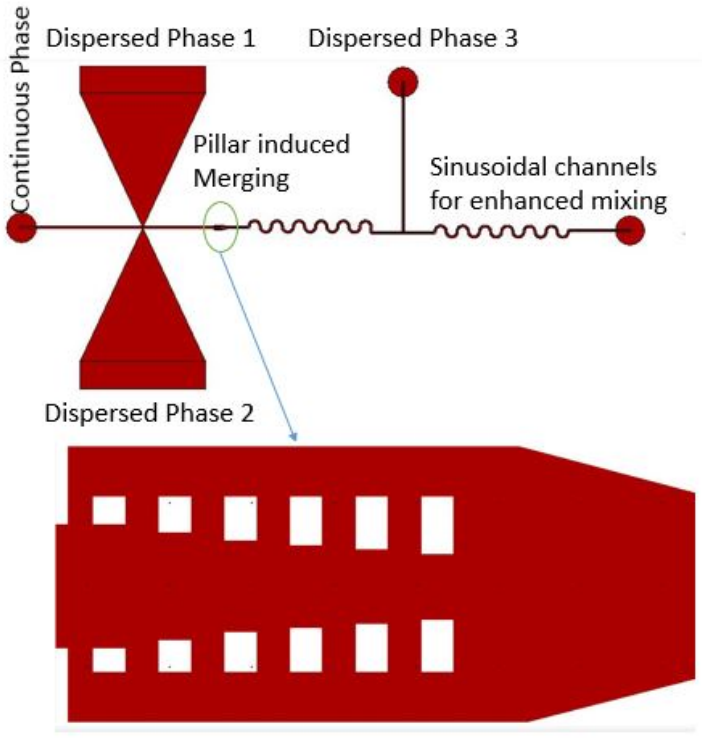

Figure 1: Schematic of the microfluidic device.

Tapered double T-junction with an angle of $25^{\circ}$ is used to generate the droplets of dispersed phases alternatively. Saqib et al. described that $25^{\circ}$ tapper has the maximum efficiency in obtaining alternating droplets where the size and spacing of droplets are also uniform [5]. Both reagents are mixed in 1 to 1 volume ratio, making it essential to use this geometry. Alternating droplets are merged downstream in pillar array using a pillared channel where these pillars force the consecutive droplets to merge due to decrease in flow speed [6]. The channel dimensions are extended from $150 \mu \mathrm{m}$ to $350 \mu \mathrm{m}$ which causes the droplets to slow down so that they can merge. Pillar structure keeps the droplets in straight line and helps the droplet to stop until the next droplet arrives and merges. The length of merging structure is optimized so that only two droplets, one from each source can merge. Sinusoidal channels enhance the mixing of two reagents in merged droplets. Finally, the third reagent (ammonia solution) is introduced to initiate the reaction and precipitates are collected at the end.

Polydimethylsiloxane (PDMS) based devices are fabricated using soft lithography technique. The $100 \mathrm{~mL}$ solution with $1.09 \mathrm{~g}$ of $\mathrm{FeCl}_{3} .6 \mathrm{H}_{2} \mathrm{O}$ and $0.4 \mathrm{~g}$ of $\mathrm{FeCl}_{2} .4 \mathrm{H}_{2} \mathrm{O}$ was used as the dispersed phase $1.0 .75 \mathrm{~g}$ of chitosan was dissolved in $100 \mathrm{~mL}$ of $0.3 \%$ acetic acid and used as dispersed phase 2. 10\% ammonia solution was 
used as dispersed phase 3 and olive oil was used as the continuous phase. Nanoparticles were collected, centrifuged and diluted in deionized water. TEM imaging and FTIR was done for the sample to characterize the nanoparticles.

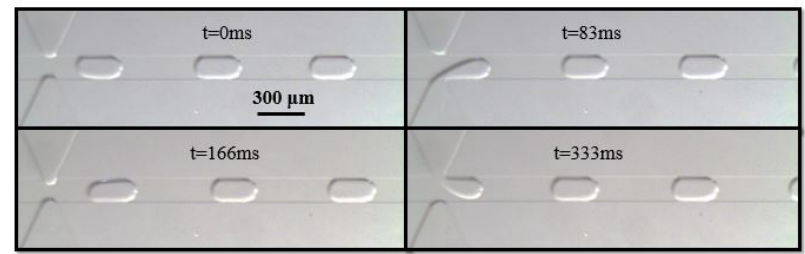

Figure 2: Alternating droplet generation using taper angle of $25^{\circ}$.

Figure 2 shows alternate droplet generation from two dispersed phases. Figure 3 illustrates the merging of consecutive droplets from different sources in pillar induced wide channel.

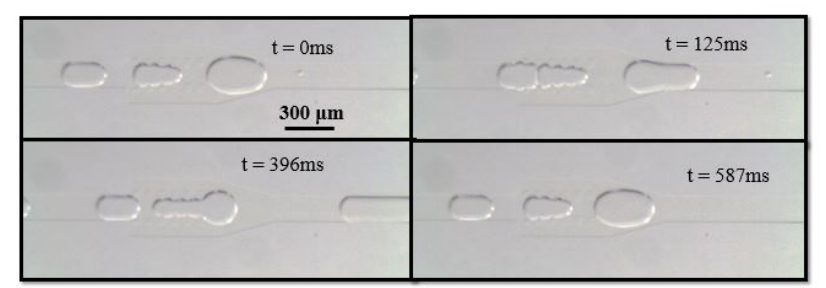

Figure 3: Merging of two alternating droplets (chitosan and iron chloride droplets) via the pillar array.

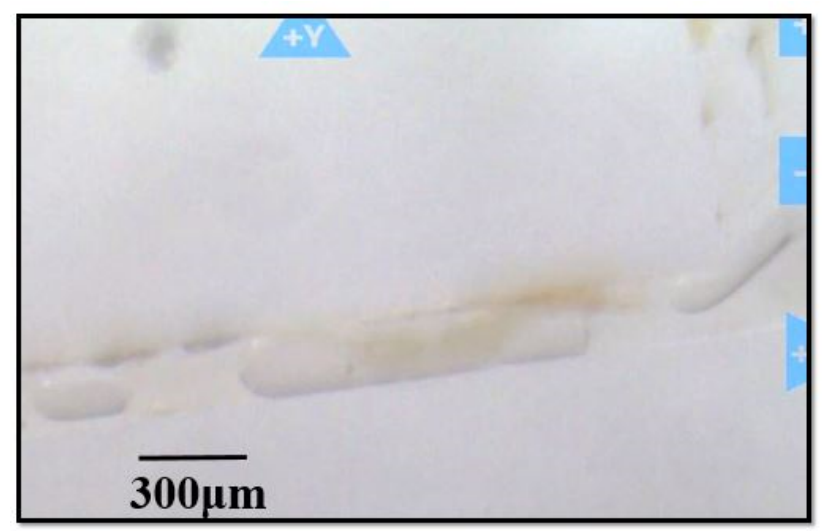

Figure 4: Merging of ammonia solution to initiate the precipitation.

Figure 4 shows the start of reaction when ammonia solution is combined with merged droplet, as color of droplet turns from transparent to yellowish color, indicating the formation of nanoparticles.

\section{RESULTS AND DISCUSSION}

TEM analysis was done to analyze the nanoparticles.

Table 1: EDX results of iron-oxide nanoparticles.

\begin{tabular}{|l|l|l|}
\hline Element & Iron & Oxygen \\
\hline Weight Percentage & 55.8 & 44.9 \\
\hline
\end{tabular}

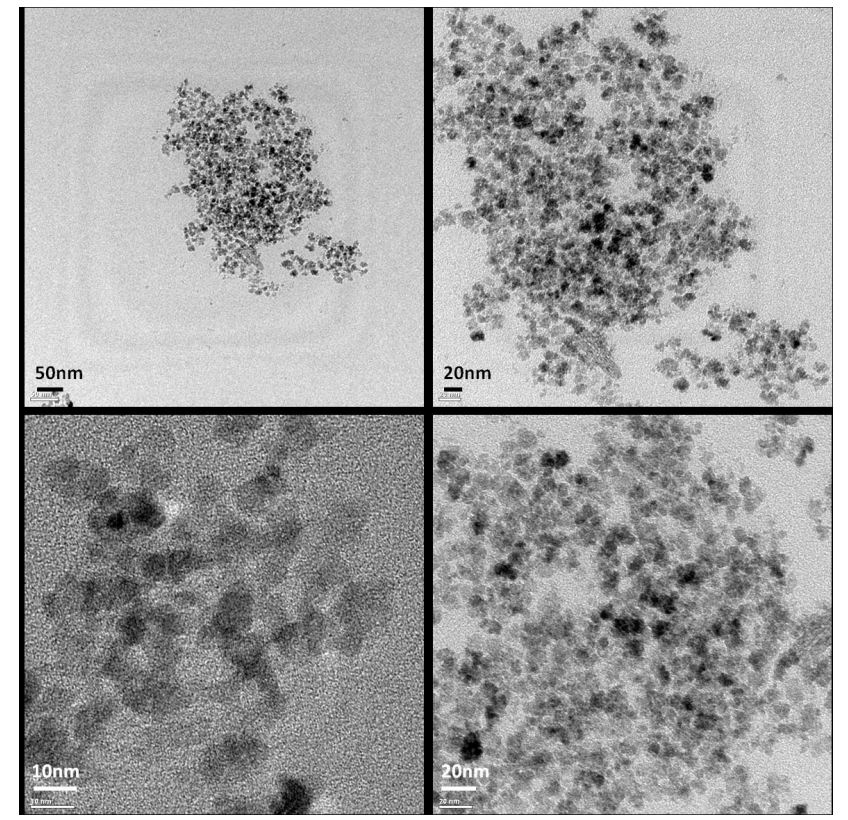

Figure 5: TEM images of synthesized nanoparticles.

Figure 5 shows TEM images of nanoparticles. According to these images, the average size of chitosan coated ironoxide nanoparticles are $8.5 \pm 1.4 \mathrm{~nm}$. Energy dispersive X-ray (EDX) data along with the TEM images shows the presence of iron-oxide nanoparticles. Compared to the results of the batch techniques, the particles produced in the microfluidic reactor had narrower size distribution [4].

In order to confirm the presence of Chitosan, FTIR was performed. FTIR data was plotted to compare the corresponding peaks and is presented in Figure 6.

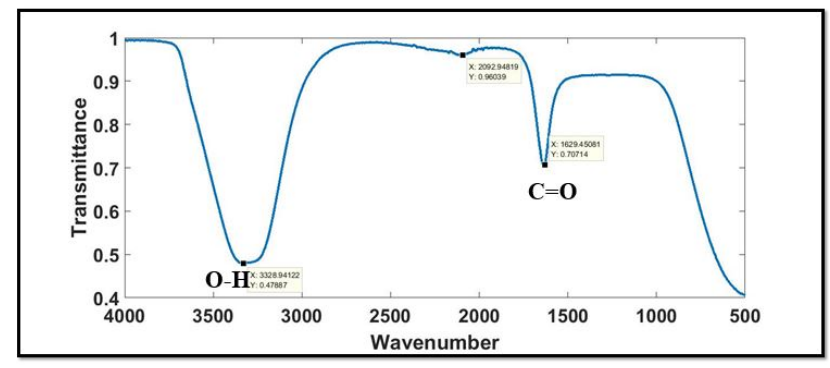

Figure 6: FTIR spectra of nanoparticles.

Chitosan presence in the nanoparticles is identified using the FTIR analysis. Successful coating of iron-oxide nanoparticles with chitosan is confirmed by the appearance of characteristic bands like $\mathrm{OH}$ at $3330 \mathrm{~cm}^{-1}$ and $\mathrm{C}=\mathrm{O}$ at $1629 \mathrm{~cm}^{-1}$ [4]. TEM results together with FTIR demonstrate the synthesis of chitosan coated ironoxide nanoparticles.

\section{CONCLUSION}

In this work synthesis of iron-oxide embedded chitosan nanoparticles have been realized for the first time using microfluidic platform which resulted in a narrower size distribution compared to the batch-wise methods 
reported in literature [4]. Residence time has been significantly reduced as compared to batch-wise synthesis.

\section{ACKNOWLEDGEMENTS}

This work was funded by the Science Academy Young Scientists Award (BAGEP 2016). We also thank Eliza Sopubekova for her assistance in FTIR and Mr. Mustafa Güler for his assistance in TEM analysis.

\section{REFERENCES}

[1] M. Hosokawa and M. Hosokawa, Nanoparticle Technology Handbook. Elsevier Science Limited, 2007.

[2] E. Y. Erdem, J. C. Cheng, F. M. Doyle, and A. P. Pisano, "Multi-Temperature Zone, Droplet-based Microreactor for Increased Temperature Control in Nanoparticle Synthesis," Small, vol. 10, no. 6, pp. 1076-1080, 2013.

[3] Y. He, M. Pei, Y. Du, F. Yu, L. Wang, and W. Guo, "Synthesis, characterization and application of chitosan coated Fe3O4particles as an adsorbent for the removal of furfural from aqueous solution," $R S C$ Advances, vol. 4, no. 57, p. 30352, 2014
[4] G. Ünsoy, S. Yalçın, R. Khodadust, G. Gündüz, and U. Gündüz, "Synthesis optimization and characterization of chitosan-coated iron oxide nanoparticles produced for biomedical applications," Journal of Nanoparticle Research, vol. 14, no. 11, 2012.

[5] M. Saqib, O. B. Şahinoğlu, and E. Y. Erdem, "Alternating Droplet Formation by using Tapered Channel Geometry," Scientific Reports, vol. 8, no. 1, 2018.

[6] X. Niu, S. Gulati, J. B. Edel, and A. J. Demello, "Pillar-induced droplet merging in microfluidic circuits," Lab on a Chip, vol. 8, no. 11, p. 1837 , 2008.

\section{CONTACT}

*Corresponding author: yeganerdem@bilkent.edu.tr 